ANNALES

POLONICI MATHEMATICI

XLVI (1985)

\title{
On G-foliations
}

\author{
by ROBERT WolaK (Kraków)
}

Franciszek Leja in memoriam

\begin{abstract}
In this short note the author looks at how properties of a pseudogroup of automorphisms of a given $G$-structure influence topological and geometrical properties of $G$ foliations modelled on this $G$-structure.
\end{abstract}

0. Introduction. All the geometrical objects considered in this paper are smooth, i.e., of $C^{\infty}$ differentiability.

Let $N$ be a $q$-manifold and $\Gamma$ a pseudogroup of diffeomorphisms of the manifold $N$. For any open subset $U$ of $N$ by $\Gamma(U)$ we denote the set $\{f \in \Gamma: \operatorname{dom} f=U\}$. We say that the pseudogroup $\Gamma$ has the property $E_{k}, k$ any integer, if for any point $x$ of the manifold the spaces $\left(j_{x}^{k} f: f \in \Gamma\left(U_{n}\right)\right\}$ are equal for some sequence of open subsets $U_{n}$ such that $U_{0}=N$ and $\cap U_{n}=\{x\}$.

EXAMPLES. 1. Any pseudogroup of diffeomorphisms generated by a group of global diffeomorphisms has the property $E_{k}$ for any $k$.

2. Let $B(N, G)$ be a regular $G$-structure on a simple connected compact manifold $N$ for a group $G$ of inite type $k$. The pseudogroup $\Gamma$ generated by the flows of infinitesimal automorphisms of this $G$-structure has the property $E_{k}$. In fact, the sheaf of germs of infinitesimal automorphisms of the $G$ structure $B(N, G)$ is constant (cf. [4]). Thus any germ of an infinitesimal automorphism can be extended to a global infinitesimal automorphism of $B(N, G)$, hence any diffeomorphism from some flow, with the domain small enough, can be extended to a global diffeomorphism, so the pseudogroup has the property $E_{k}$.

Let $G$ be a closed subgroup of the linear group $\operatorname{GL}(q)$ of finite type $k$, let $B(N, G)$ be a $G$-structure on the manifold $N$. Let $\Gamma$ be a pseudogroup of automorphisms of the $G$-structure $B(N, G)$ having the property $E_{k}$. Let $F$ be a $\Gamma$-foliation on an $n$-manifold $M$ in the sense of Haefliger (cf. [5]). Then we have the following

THEOREM 1. The lifted foliation $\tilde{F} \cdot$ to the universal covering $\tilde{M}$ of the manifold $M$ is simple, i.e., defined by a global submersion. 
THEOREM 2. Assume that the manifold $M$ is compact. The growth of the leaves of the foliation $F$ is dominated by the growth of the fundamental group $\pi_{1}(M)$ of the manifold $M$.

THEOREM 3. The leaves of a complete foliation $F$ have the common universal covering space. The space of leaves of the foliation $F$ is homeomorphic to the orbit space of some action of a group on a covering space of the manifold $N$.

We would like to express our gratitude to Robert A. Blumenthal for providing the preprints of papers of his which have been the inspiration of this note.

1. G-foliations. Let $F$ be a $G$-foliation on the manifold $M$ (cf. [2]). Then the normal bundle $N(M, F)$ of the foliation $F$ admits a reduction of the structure group to the group $G$. Denote by $B(M, G ; F)$ the reduction to the group $G$ of the linear frame bundle $L(M ; F)$ of the normal bundle $N(M ; F)$.

On $L(M ; F)$ and $B(M, G ; F)$ we define an $R^{q}$-valued 1 -form $\theta$, the fundamental form, as follows (cf. [7], [8]):

$$
T_{p}(L(M ; F)) \stackrel{d \pi}{\longrightarrow} T_{n p} M \rightarrow N_{\pi p}(M ; F) \stackrel{p^{-1}}{\longrightarrow} R^{q},
$$

where $\pi: L(M ; F) \rightarrow M$ is the natural projection. Then

$$
R_{a}^{*} \theta=a^{-1} \theta \quad \text { for any } a \in G,
$$

and

$$
L_{A^{*}} \theta=-A \cdot \theta \quad \text { for any } A \in \operatorname{Lie}(G)=\text {. }
$$

On the manifold $B(M, G ; F)$ one defines a foliation $F_{1}$ of dimension $n-q$ as follows:

$$
F_{1}=\left\{X \in T B(M, G ; F): i_{X} \theta=0, i_{X} d \theta=0\right\}
$$

(cf. [7], [8]).

Let us choose a splitting $s$ of the exact sequence

$$
0 \rightarrow F \rightarrow T M \stackrel{s}{\leftrightarrows} N(M, F) \rightarrow 0 .
$$

Take any $q$-dimensional subspace $H_{p}$ at a point $p$ in $T_{p} B(M, G ; F)$ such that its projection $d \pi H_{p}$ onto $T_{\pi p} M$ is equal to $s\left(N_{\pi p}(M, F)\right)$. From now on we shall consider only such subspaces and call them the horizontal subspaces.

Let us take two horizontal subspaces $H_{1}$ and $H_{2}$ at a point $p$ of $B(M, G ; F)$. Take any vector $v$ of $R^{q}$. Then there exist the unique vectors $X_{1} \in H_{1}$ and $X_{2} \in H_{2}$ such that $\theta\left(X_{1}\right)=\theta\left(X_{2}\right)=v$. Thus $\theta\left(X_{1}-X_{2}\right)=0$ and $d \pi\left(X_{1}-X_{2}\right)=0$. Therefore $X_{1}-X_{2}=A^{*}$ for some vector $A \in g$. In this way, for any two horizontal subspaces $H_{1}$ and $H_{2}$ at a point $p$ we define a linear mapping $S_{H_{1} H_{2}}: R^{q} \rightarrow g$ by putting $S_{H_{1} H_{2}}(v)=A$ such that if $X_{1} \in H_{1}$, $X_{2} \in H_{2}$ and $\theta\left(X_{1}\right)=\theta\left(X_{2}\right)=v$, then $X_{1}-X_{2}=A^{*}$. 
If a linear mapping $S: R^{q} \rightarrow g$ and a horizontal space $H$ at a point $p$ are given we define another horizontal space $H^{\prime}$ at $p$ in the following way:

$$
H^{\prime}=\left\{X^{\prime}=X+S(v)^{*}: \theta(X)=v, X \in H\right\} .
$$

Then

$$
X^{\prime}-X=S(v)^{*} \quad \text { and } \quad S_{H^{\prime} H}=S .
$$

For any horizontal subspace $H$ we can define the following mapping $c_{H}: R^{q} \wedge R^{q} \rightarrow R^{q}$.

$$
c_{H}(u \wedge v)=\langle X \wedge Y, d \theta\rangle \quad \text { for } X ; Y \in H \text { such that } \theta(X)=u, \theta(Y)=v \text {. }
$$

Then comparing the mappings $c_{H}$ and $c_{H^{\prime}}$ defined for two different horizontal subspaces $H$ and $H^{\prime}$ at a given point $p$ we have the following:

$$
\begin{aligned}
c_{H}(u \wedge v)-c_{H^{\prime}}(u \wedge v) & =\langle X \wedge Y, d \theta\rangle-\left\langle X^{\prime} \wedge Y^{\prime}, d \theta\right\rangle \\
& =\left\langle\left(X-X^{\prime}\right) \wedge Y, d \theta\right\rangle-\left\langle X^{\prime} \wedge\left(Y^{\prime}-Y\right), d \theta\right\rangle \\
& =\left\langle S_{H H^{\prime}}(u)^{*} \wedge Y, d \theta\right\rangle-\left\langle X^{\prime} \wedge S_{H^{\prime} H}(v)^{*}, d \theta\right\rangle \\
& =-\frac{1}{2}\left(S_{H H^{\prime}}(u) \cdot \theta(Y)+S_{H^{\prime} H}(v) \cdot \theta(X)\right) \\
& =\frac{1}{2}\left(S_{H^{\prime} H}(u)(v)-S_{H^{\prime} H}(v)(u)\right)=\partial S_{H^{\prime} H}(u \wedge v),
\end{aligned}
$$

where $\partial$ is the antisymmetrization operator.

Therefore for any $p \in B(M, G ; F)$ the mappings $c_{H}$ define the unique class $c(p)$ in $\operatorname{Hom}\left(R^{q} \wedge R^{q}, R^{q}\right) / \partial \operatorname{Hom}\left(R^{q}, \underline{g}\right)$. We call this tensor the structure tensor of the transverse $G$-structure $B(M, G ; F)$. Since $\partial \operatorname{Hom}\left(R^{q} ; g\right)$ is a vector subspace of $\operatorname{Hom}\left(R^{q} \wedge R^{q}, R^{q}\right)$, we can choose a supplementary subspace $C$ to $\partial \operatorname{Hom}\left(R^{q}, g\right)$ in $\operatorname{Hom}\left(R^{q} \wedge R^{q}, R^{q}\right)$. This choice, at a point $p$ of $B(M, G ; F)$, distinguishes a family of horizontal subspaces $H$ such that $c_{H} \in C$. If $H_{1}$ and $H_{2}$ are two such horizontal subspaces at a point $p$

so

$$
c_{H_{1}}(u \wedge v)-c_{H_{2}}(u \wedge v)=\partial S_{H_{2} H_{1}}(u \wedge v)=0,
$$

$$
S_{H_{1} H_{2}} \in g^{(1)}-g^{(1)} \quad \text { the first prolongation of the Lie algebra } \underline{g} \text {. }
$$

Thus the choice of $C$ defines a $G^{(1)}$-structure on $L\left(B(M, G ; F), F_{1}\right)$, where

$$
G^{(1)}=\left\{\left(\begin{array}{ll}
\text { id } & 0 \\
h & \text { id }
\end{array}\right) \in \operatorname{GL}\left(R^{q}+\underline{g}\right): h \in g^{(1)}\right\} .
$$

We call this $G^{(1)}$-structure the first prolongation of the transverse $G$-structure $B(M, G ; F)$ and denote it by $B^{1}(M, G ; F)$. By induction, we define further prolongations, i.e., $B^{k+1}(M, G ; F)$ is the first prolongation of the transverse $G^{k}$-structure $B^{k}(M, G ; F)$. If the group $G$ is of finite type $k$, after a finite number $k$ of steps, we get an $\{e\}$-structure $B^{k}(M, G ; F)$, so the foliation $F_{k}$ is transversely parallelisable. 
Remark. Let a transverse $G$-structure be projectible (cf. [7], [8]). With a suitable choice of the splitting, if the diagram

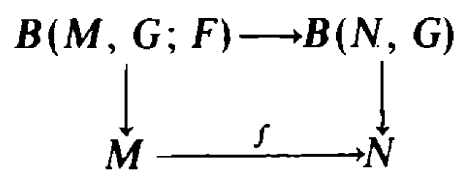

is commutative, we have the following equality for the structure tensors: $f^{*} c_{N}=c_{M}$.

2. Proof of Theorem 1. The proof is based on the following lemma:

LeMma 1. Let $G$ be a closed subgroup of the group $\mathrm{GL}(q)$ of finite type and let $\Gamma$ be a pseudogroup of automorphisms of a $G$-structure $B(N, G)$. Then the restriction mapping $\Gamma\left(U_{m}\right) \rightarrow \Gamma\left(U_{m+1}\right)$ is bijective. In particular, any automorphism of the G-structure belonging to $\Gamma$ can be extended to a global one.

Proof. Let $f \in \Gamma\left(U_{m+1}\right)$. Then the lift $\bar{f}$ to $B^{k}(N, G)$ preserves the $\{e\}-$ structure on $B^{k}(N, G)$, i.e., the parallelism $\left\{X_{1}, \ldots, X_{r}\right\}$ of $B^{k}(N, G)$ (cf. [10]). Thus

$$
\bar{f} \exp t X_{i}=\exp t X_{i} \bar{f} \quad \text { for any } t \text { and } i=1, \ldots, r
$$

whenever $\exp t X_{i}$ is defined.

Let $g$ be an element of $\Gamma\left(U_{m}\right)$ such that $j_{x}^{k} g=j_{x}^{k} f$. Then for the lifted mapping $\bar{g}$ to $B^{k}(N, G)$ we have the equality $\bar{g}(p)=\bar{f}(p)$ for any $p$ over $x$. The set $A=\left\{p \in B^{k}(N, G): \bar{f}(p)=\bar{g}(p)\right\}$ is nonempty and closed. It is open as well because of $(*)$. The uniqueness is proved in the same way.

Let $\left\{U_{i}, f_{i}, g_{i j}\right\}$ be a $\Gamma$-cocycle defining the given $\Gamma$-foliation. For each $g_{i j}$, by Lemma 1 , there exists the unique global diffeomorphism $\bar{g}_{i j}$ of the pseudogroup $\Gamma$ extending $g_{i j}$.

Let $\underline{H}=\left\{\left(L_{g} f_{i}\right)_{x}\right.$ for $x \in U_{i}, g \in \Gamma_{j}$, where ()$_{x}$ denotes the germ at $x$. The space $\underline{H} \rightarrow M$ with the natural projection $w$ and the sheaf topology admits a $C^{x}$-manifold structure of dimension $n$. Any connected component of $\underline{H}$ is a cover space of $\boldsymbol{M}$. Let us take such a component $\hat{M}$. Then there exists a natural equivariant submersion, denoted by $f$, of $\hat{M}$ into $N$

$$
\hat{M} \ni\left(L_{g} f_{i}\right)_{x} \mapsto g f_{i}(x) \in N .
$$

The lifted foliation $\hat{F}$ to $\hat{M}$ is just the foliation defined by this submersion.

3. Proof of Theorem 2. Lemma 1 and the way the space $H$ has been constructed allows us to use precisely the methods of R. Blumenthal (cf. [1]). We leave it to the reader to fill the details.

\section{Complete $G$-foliations.}

Definition. Let $G$ be a closed subgroup of $\mathrm{GL}(q)$ of finite type $k$. We say that a $G$-foliation is complete if the transverse parallelism of $B^{k}(M, G ; F)$ is complete and foliation preserving. 
LeMma 2. Let the $k$-th structure tensor of $B(N, G)$ be zero, and let the transverse parallelism be complete. Then the $G$-foliation $F$ is complete.

Proof. Since $\left(g^{(k-1)}\right)^{(1)}=g^{(k)}=0$, the connections are determined by their torsion tensors (cf. [4]). Therefore the torsion-free connection on $B^{k-1}(N, G)$ is unique, and therefore it determines a basic connection on $B^{k-1}(M, G ; F)$. Thus the fundamental horizontal and vertical vector fields on $B^{k-1}(M, G ; F)$ define a transverse parallelism. One can easily prove, using the same methods as in [7], that they are infinitesimal automorphisms of the foliation $F_{k}$.

Proof of Theorem 3. By Theorem 1 there exists a cover $\hat{M}$ of the manifold $M$ such that the lifted foliation $\hat{F}$ is defined by a global submersion $f: \hat{M} \rightarrow N$. Thus the foliation $\hat{F}_{k}$ of $B^{k-1}(\hat{M}, G ; \hat{F})$ is defined by the submersion $f^{k}$

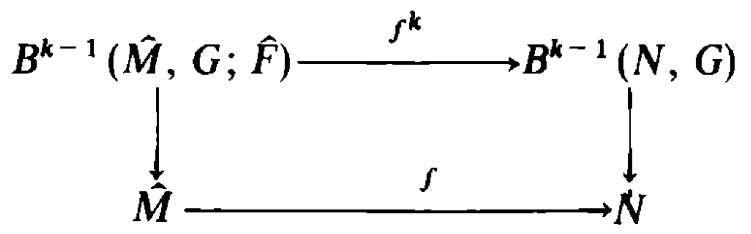

The space $B^{k-1}(\hat{M}, G ; \hat{F})$ is a cover space of $B^{k-1}(M, G ; F)$ and the foliation $\hat{F}_{k}$ is the lifted foliation of $F_{k}$. The foliation $\hat{F}_{k}$ has closed leaves and is transversely parallelisable by its global infinitesimal automorphisms, since the foliation $F_{k}$ is. These vector fields are complete as well. By Lemma 1 of [7] $B^{k-1}(\hat{M}, G ; \hat{F})$ is a locally trivial fibre bundle over the Hausdorff manifold $B^{k-1}(\hat{M}, G ; \hat{F}) / \hat{F}_{k}$. Thus the leaves of the foliation $F$ have a common universal covering space.

Since the transverse parallelism of $\hat{F}_{k}$ is by infinitesimal automorphisms, it projects to a complete parallelism of $B^{k-1}(\hat{M}, G ; \hat{F}) / \hat{F}_{k}$ and is mapped by the induced mapping $\bar{f}_{k}$ onto the parallelism of $B^{k-1}(N, G)$. On each manifold $B^{k-1}(N, G)$ and $B^{k-1}(\hat{M}, G ; \hat{F}) / \hat{F}_{k}$ we define a connection which preserves the parallelism. Applying Theorem 3 of [6] we obtain that the mapping $\bar{f}_{k}: B^{k-1}(\hat{M}, G ; \hat{F}) / \hat{F}_{k} \rightarrow B^{k-1}(N, G)$ is a covering mapping. Therefore we have the following commutative diagram

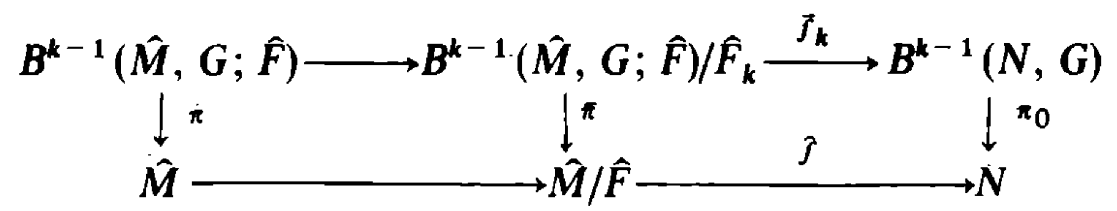

where $\hat{M} / \hat{F}$ is a $T_{1}$ manifold according to Palais [9]. Hence the mapping $\hat{f}$ is a local homeomorphism. The next step is to show that $\hat{f}$ is a covering. To prove this we need only to show that $\hat{f}$ has the property of lifting of curves. Since $B^{k-1}(N, G) \rightarrow N$ is a principal fibre bundle, for any curve $\gamma$ in $N$ there is a horizontal lift $\tilde{\gamma}$ of the curve $\gamma$ to $B^{k-1}(N, G)$. Because $\overline{f_{k}}$ is a covering mapping, the curve $\tilde{\gamma}$ can be lifted to a curve $\bar{\gamma}$ in $B^{k-1}(\hat{M}, G ; \hat{F}) / \hat{F}_{k}$. Thus 
the curve $\overline{\pi \gamma}$ is a lift of $\gamma$ to $\hat{M} / \hat{F}$. The choice of a point $y$ in $\hat{f}^{-1}(\gamma(0))$ forces the following choices in the two liftings executed: $\bar{\pi}^{-1}(y) \ni \vec{y}$ and $\bar{f}_{k}(\bar{y}) \in \pi_{0}^{-1}(\gamma(0))$. Therefore the manifold $\hat{M} / \hat{F}$ is a Hausdorff manifold. We denote the manifold $\hat{M} / \hat{F}$ by $\hat{N}$.

Since the mapping $\hat{f}$ is a covering mapping, we can lift the pseudogroup $\Gamma$ to $\hat{N}$ - denote the lifted pseudogroup by $\hat{\Gamma}$. If the pseudogroup $\Gamma$ has the property $E_{k}$, then the lifted pseudogroup has this property as well. The foliation $F$ can be considered as a $\hat{\Gamma}$-foliation.

Let us choose a point $x_{0}$ of the manifold $M$. Any loop at $x_{0}$ defines an element of $\hat{\Gamma}(\hat{N})$ in the following way. The loop $\gamma$ can be covered by a finite number of sets $U_{i_{1}}, \ldots, U_{i_{s}}, U_{i_{1}}=U_{i_{s}}$. We can choose a sequence of numbers $t_{i} \in[0,1], i=0, \ldots, s$, such that $t_{0}=0, \gamma\left(t_{i}\right) \in U_{i_{i}} \cap U_{i_{i+1}}, i=1, \ldots, s-1$, $t_{s}=1$. Then

$$
f_{i_{1}}\left(\gamma\left(t_{1}\right)\right)=g_{i_{1} i_{2}} f_{i_{2}}\left(\gamma\left(t_{1}\right)\right)
$$

Because of the uniqueness of the extension of $g_{i_{1} i_{2}}$, the choice of the global automorphisms $g_{i_{1} i_{2}}$ does not depend on a choice of $t_{1}$. Doing it step by step we get

Thus

$$
f_{i_{j}}\left(\gamma\left(t_{j}\right)\right)=g_{i_{j} i_{j+1}} f_{i_{j+1}}\left(\gamma\left(t_{j}\right)\right) .
$$

$$
g_{i_{1} i_{2}} \ldots g_{i_{j-1} i_{j}} f_{i_{j}}\left(\gamma\left(t_{j}\right)\right)=g_{i_{1} i_{2}} \ldots g_{i_{j} i_{j+1}} f_{i_{j+1}}\left(\gamma\left(t_{j}\right)\right) \text {, }
$$

and the curve $\tilde{\gamma}: t \rightarrow\left(g_{i_{1} i_{2}} \ldots g_{i_{j-1} i_{j}} f_{i_{j}}\right)(\gamma(t)), t \in\left[t_{j-1}, t_{j}\right]$, is the lift of the curve $\gamma$ to $\underline{H}$ at $\left(f_{i_{1}}\right)(\gamma(0))$. Therefore $\tilde{\gamma}(1)=\left(g_{i_{1} i_{2}} \ldots g_{i_{s_{1}} i_{1}} f_{i_{1}}\right)(\gamma(1))$. If $\gamma^{\prime}$ is a loop at $x_{0}$ homotopic to $\gamma$ we get

$$
\left(g_{i_{1}^{\prime} i_{2}^{\prime}}^{\prime} \ldots g_{i_{i}^{\prime} i_{1}}\right)\left(f_{i_{1}}\left(x_{0}\right)\right)=\left(g_{i_{1} i_{2}} \ldots g_{i_{s} i_{1}}\right)\left(f_{i_{1}}\left(x_{0}\right)\right) \text {. }
$$

By Lemma 1, $g_{i_{1}^{\prime} i_{2}^{\prime}} \ldots g_{i_{i}^{\prime} i_{1}^{\prime}}=g_{i_{1} i_{2}} \ldots g_{i_{s} i_{1}}$. Thus the correspondence defined above induces a homomorphisms $h$ of the groups

$$
h: \pi_{1}\left(M, x_{0}\right) \rightarrow \operatorname{Aut}(B(\hat{N}, G))
$$

which is defined up to conjugation.

If we denote the im $h$ by $K$, using the standard argument (cf. [1], [3]) we can show that the space of leaves of the foliation $F$ is homeomorphic to the space $\hat{N} / K$.

Corollary. Let $L$ be a leaf of the foliation $F$. Denote the corresponding orbit on $\hat{N}$ by $K_{L}$. Then

(i) $L$ is proper iff $K_{L}$ is discrete,

(ii) $L$ is closed iff $K_{L}$ is discrete and closed,

(iii) $L$ is dense iff $K_{L}$ is dense.

The proof is straightforward. 


\section{References}

[1] R. A. Blumentha1, Transversely homogeneous foliations, Ann. Inst. Fourier 29, 4 (1979), $143-158$.

[2] T. Duchamp, Characteristic invariants of G-foliations, Ph. D. thesis, University of Illinois, Urbana 1976.

[3] E. Fedida, Sur les feuilletages de Lie, C. R. Acad. Sci. Paris 272 (1971), 999-1002.

[4] A. F ujimoto, Theory of G-structures, Publications of the Study Group of Geometry, vol. 1, Tokyo 1972.

[5] A. Haefliger, Feuilletages sur les variétés ouvertes, Topology 9 (1970), 183-194.

[6] N. Hicks, A theorem on affine connections, Ill. J. Math. 3 (1959), 242-254.

[7] P. Molino, Géométrie globale des feuilletages riemanniens, Proc. Kon. Nederland Acad, Ser. A, 1, 85 (1982), 45-76.

[8] -, Feuilletages riemanniens, Université des Sciences et Techniques du Languedoc, Institut de Mathématiques, D.E.A. de Mathématiques, Option Géométrie Différentielle 19821983.

[9] R. Palais, The Lie Theory of Transformation Groups, Memoirs Amer. Math. Soc. 22 (1957).

[10] I. M. Singer, S. Sternberg, The infinite groups of Lie and Cartan, J. Analyse Math. 15 (1965), 1-114.

INSTYTUT MATEMATYKI

I NIWERSYTETU JAGIELLON'SKIEGO

KRAKÓW 